\title{
GC1118, a novel anti-EGFR antibody, has potent KRAS mutation- independent antitumor activity compared with cetuximab in gastric cancer
}

\author{
Ji Eun Park ${ }^{1}$ Mei Hua Jin ${ }^{1} \cdot$ Minkyu Hur ${ }^{3} \cdot$ Ah-Rong Nam ${ }^{1}$. Ju-Hee Bang ${ }^{1}$ Jonghwa Won ${ }^{3}$. Do-Youn Oh ${ }^{1,2}$. \\ Yung-Jue Bang ${ }^{1,2}$
}

Received: 26 July 2018 / Accepted: 23 February 2019 / Published online: 27 February 2019

(c) The International Gastric Cancer Association and The Japanese Gastric Cancer Association 2019

\begin{abstract}
Background EGFR overexpression in gastric cancer (GC) has been reported in about $30 \%$ of patients. However, the antiEGFR antibodies cetuximab and panitumumab have failed to improve overall survival of GC patients in combination with cytotoxic chemotherapy compared with chemotherapy alone. GC1118, a novel anti-EGFR antibody with a distinct binding epitope compared with cetuximab or panitumumab, has not been tested in GC.

Methods GC cell lines, SNU-1, SNU-5, SNU-16, SNU-216, SNU-484, SNU-601, SNU-620, SNU-638, SNU-668, SNU-719, AGS, MKN-45, NCI-N87, and KATO-III, were employed to test the effect of cetuximab or GC1118 alone, and combined with the cytotoxic agent cisplatin or 5-fluorouracil (5-FU). Cells were also treat with or without high-affinity ligands EGF $20 \mathrm{ng} / \mathrm{ml}$ or HB-EGF $100 \mathrm{ng} / \mathrm{ml}$.

Results GC1118 exhibited a more potent growth inhibition effect in the majority of cell lines than cetuximab in MTT assay, regardless of the KRAS mutation status of cell lines. Co-treatment of GC1118 and cisplatin or 5-FU inhibited colony formation and migration to a greater extent, even following EGFR ligand stimulation. Ligand-induced p-AKT and p-ERK upregulation were more potently inhibited by combination treatment with GC1118 and chemotherapeutic agents compared with cetuximab plus chemotherapeutic agents. GC1118 also showed more potent anti-tumor effects compared with cetuximab in a mouse xenograft model.

Conclusion Taken together, GC1118 alone or in combination with cytotoxic chemotherapeutic agents exerted more potent anti-tumor effects than cetuximab in GC cells, regardless of $K R A S$ status. These findings support the further clinical development of GC1118 for the treatment of GC.
\end{abstract}

Keywords EGFR $\cdot$ GC1118 $\cdot$ Cetuximab $\cdot$ Gastric cancer $\cdot$ KRAS

Ji Eun Park and Mei Hua Jin contributed equally to this work.

Do-Youn Oh

ohdoyoun@snu.ac.kr

1 Cancer Research Institute, Seoul National University College of Medicine, Seoul, South Korea

2 Department of Internal Medicine, Seoul National University College of Medicine, 101 Daehak-ro, Jongno-gu, Seoul 03080, South Korea

3 MOGAM Institute for Biomedical Research, Yongin, Gyeonggi-do, South Korea

\section{Introduction}

The epidermal growth factor receptor (EGFR) is a transmembrane receptor and is one of the most commonly expressed oncogenes in solid tumors [1,2]. Given that the EGFR pathway has significant implications for carcinogenesis, including the survival, migration, angiogenesis, differentiation, and apoptosis of cancer cells, research on the targeted inhibition of EGFR has been in progress in recent decades, with success in several tumor types including colon cancer and head and neck cancer [1-3]. In colon cancer, anti-EGFR treatment is applicable only for $K R A S$ wild-type tumors. In gastric cancer (GC), EGFR overexpression and/ or amplification is observed in about $30 \%$ of cases and is associated with poor prognosis [4]. However, the anti-EGFR 
antibodies, cetuximab and panitumumab, have failed to improve the overall survival of GC patients when administered in combination with cytotoxic chemotherapy compared with chemotherapy alone $[5,6]$.

GC1118, a novel EGFR monoclonal antibody, exhibits a potent anti-tumor effect in models of colorectal cancer (CRC) [7]. Compared with cetuximab and panitumumab, GC1118 possesses a distinct binding epitope and GC1118 has binding affinity of $0.16 \mathrm{nmol} / \mathrm{L}\left(K_{\mathrm{D}}\right)$ to EGFR, whereas cetuximab and panitumumab have the $K_{\mathrm{D}}$ of $4.5 \mathrm{nmol} / \mathrm{L}$ and $0.05 \mathrm{nmol} / \mathrm{L}$, respectively [7]. In addition, GC1118 shows more pronounced inhibitory activity in CRC [7]. Interestingly, the antitumor effects of GC1118 are observed even in KRAS mutant CRC cells [7]. In advanced GC, the incidence of KRAS mutation and amplification is about $5 \%$ and $9 \%$, respectively $[8,9]$.

The purpose of our study was to investigate the antitumor efficacy of GC1118 compared with cetuximab in GC. Furthermore, we evaluated the synergistic efficacy of GC1118 when administered in combination with cytotoxic chemotherapies following stimulation with EGFR ligands.

\section{Materials and methods}

\section{Human cell lines and reagents}

A total of 14 human GC cell lines were used in our study. SNU-1, SNU-5, SNU-16, SNU-216, SNU-484, SNU601, SNU-620, SNU-638, SNU-668, SNU-719, AGS, MKN-45, NCI-N87, and KATO-Ш were purchased from the Korean Cell Line Bank (Seoul, Korea). All cells were passaged less than 2 months before use and maintained in RPMI-1640 media containing 10\% fetal bovine serum (FBS; Welgene Inc., Gyeongsan, Korea) and $10 \mu \mathrm{g} / \mathrm{mL}$ gentamicin in a humidified atmosphere containing $5 \% \mathrm{CO}_{2}$ at $37^{\circ} \mathrm{C}$. GC1118 (GC Pharma, Yongin, Korea) and cetuximab (Merck, Germany) were kindly provided by MOGAM Institute for Biomedical Research (Yongin, Korea). Cisplatin and 5-fluorouracil (5-FU) were purchased from JW Pharmaceutical Co. (Seoul, Korea) and Ildong Pharmaceutical Co. (Seoul, Korea), respectively.

\section{Cell growth assay}

Cells were seeded into 96-well plates and exposed to increasing concentrations of cetuximab $(0,0.01,0.1,1$, 10 , or $100 \mu \mathrm{g} / \mathrm{mL})$ or $\mathrm{GC} 1118(0,0.001,0.01,0.1,1,10$, 100 , or $1000 \mu \mathrm{g} / \mathrm{mL}$ ) for 1,3 or 5 days. Next, $50 \mu \mathrm{L}$ of 3-(4,5-dimethylthiazol-2yl)-2,5-diphenyltetrazolium bromide (MTT) solution (Sigma-Aldrich, St. Louis, MO, USA) was added to each well and plates were incubated at $37{ }^{\circ} \mathrm{C}$ for $4 \mathrm{~h}$. The culture medium was removed and $150 \mu \mathrm{L}$ of
DMSO was added to each well. Cell viability was measured at $540 \mathrm{~nm}$ using a VersaMax Microplate Reader (Molecular Devices). The experiments were performed in triplicate.

\section{Colony-forming assay}

Cells were seeded into 6-well plates and exposed to indicate concentrations of GC1118 or cytotoxic agents (cisplatin or $5-\mathrm{FU}$ at $0.01,0.1$, or $1 \mu \mathrm{g} / \mathrm{mL}$ ) or their combination. After 10 days of incubation, the colonies were stained with Coomassie blue for $2 \mathrm{~h}$ and counted using Gel Doc system software (Bio-Rad, Hercules, CA, USA). Each experiment was repeated three times.

\section{Western blot analysis}

Cells were seeded into 60-mm dishes and incubated for $24 \mathrm{~h}$ with serum-free media, then treated with GC1118 $(0.05,0.5$, 5 , and $50 \mu \mathrm{g} / \mathrm{mL})$ or cetuximab $(0.05,0.5,5$, and $50 \mu \mathrm{g} / \mathrm{mL})$ for $2 \mathrm{~h}$, followed by stimulation with EGF $(20 \mathrm{ng} / \mathrm{ml})$ or HBEGF $(100 \mathrm{ng} / \mathrm{ml})$ for $15 \mathrm{~min}$. Then cisplatin $(1 \mu \mathrm{g} / \mathrm{mL})$ or 5 -FU $(1 \mu \mathrm{g} / \mathrm{mL})$ was treated for $24 \mathrm{~h}$. The treatment combinations were as follows: cetuximab/GC1118 +EGF, cetuximab/GC1118 + HB-EGF, cetuximab/GC1118 + cisplatin, cetuximab/GC1118 + 5-FU, cetuximab/GC1118 + cisplatin + EGF/HB-EGF, and cetuximab/GC1118+5-FU +EGF/ HB-EGF. The treated cells were harvested and lysed on ice for $30 \mathrm{~min}$ in RIPA buffer containing protease inhibitors. Protein was extracted and equal amounts of protein were used for western blot analysis with the following primary antibodies purchased from Cell Signaling Technology (Beverley, MA, USA): phosphorylated EGFR-Tyr992 (\#2235); phosphorylated AKT-Ser473 (\#9271); phosphorylated ERK-Thr202/Tyr204 (\#9101); PARP (\#9532); and caspase-7 (\#9492). Anti- $\alpha$-Tubulin (\#T5168) and $\beta$-actin antibodies were purchased from Sigma-Aldrich. Appropriate secondary antibodies were acquired from Thermo Science Inc..

\section{Migration assay}

Cells were seeded into 6-well plates and incubated at $37^{\circ} \mathrm{C}$. After $24 \mathrm{~h}$, cells were scratched with a $200-\mu \mathrm{l}$ pipet tip and treated with GC1118 $(10 \mu \mathrm{g} / \mathrm{ml})$, cisplatin $(0.01$ and $0.1 \mu \mathrm{g} /$ $\mathrm{ml}) / 5$-FU $(0.01,0.1$, and $1 \mu \mathrm{g} / \mathrm{ml})$, or with combinations of these agents. Images were analyzed using ImageJ software at 0 and $48 \mathrm{~h}$. All experiments were performed in triplicate.

\section{ELISA}

Cells were seeded onto 6-well plates and incubated for $72 \mathrm{~h}$ before culture supernatants were collected for ELISA to determine ligand release. ELISA kits to detect the human 
EGFR ligands EGF, HB-EGF, TGF- $\alpha$, and amphiregulin were purchased from Ray Biotech, Inc..

\section{In vivo study}

Animal experiments were approved by the Institutional Animal Care and Use Committee of GC Pharma and the MOGAM Institute for Biomedical Research (Yongin, Korea). Seven-week-old female athymic nude mice were purchased from Orient Bio Inc. (Korea). AGS xenograft model mice were established by the subcutaneous inoculation of $1 \times 10^{7}$ cells in $100 \mu \mathrm{L}$ of PBS plus Matrigel (BD Bioscience, no. 356231). When the tumor volume reached $200 \mathrm{~mm}^{3}$, mice were randomly divided into three groups of six. Cetuximab or GC1118 was administered intraperitoneally twice a week at $1 \mathrm{mg} / \mathrm{kg}$ for 5 weeks, and a control group was treated with PBS. Body weight and tumor size were measured every 2-4 days. Tumor volume was calculated using the formula: tumor volume $=\left[(\text { width })^{2} \times\right.$ height] $/ 2$.

\section{Statistical analysis}

Statistical analyses were conducted using SigmaPlot version 10.0 (Systat Software Inc., San Jose, CA, USA). Experimental data are presented as mean \pm standard error
(SE). All statistical tests were two-sided. Differences were considered statistically significant for values of $p<0.05$.

\section{Results}

\section{Anti-proliferative effects of GC1118 and cetuximab in GC cells}

To evaluate the growth-inhibitory activity of GC1118 on GC cells, an MTT assay was performed. Cell growth was suppressed in the majority of cell lines treated with GC1118, particularly in the MKN-45 cell line. In contrast, no growth-inhibitory effect was observed in the SNU-484 and KATO-III cell lines (Fig. 1a).

To compare the anti-tumor effects of GC1118 with those of cetuximab, we conducted an MTT assay by comparing cell numbers on days 1, 3, and 5. As a result, we found that the anti-growth inhibitory effect of GC1118 was more obviously in KRAS wild-type cell lines, SNU-719 and MKN-45, but also in KRAS mutant SNU-601 cells $(p<0.05$, Fig. 1b).
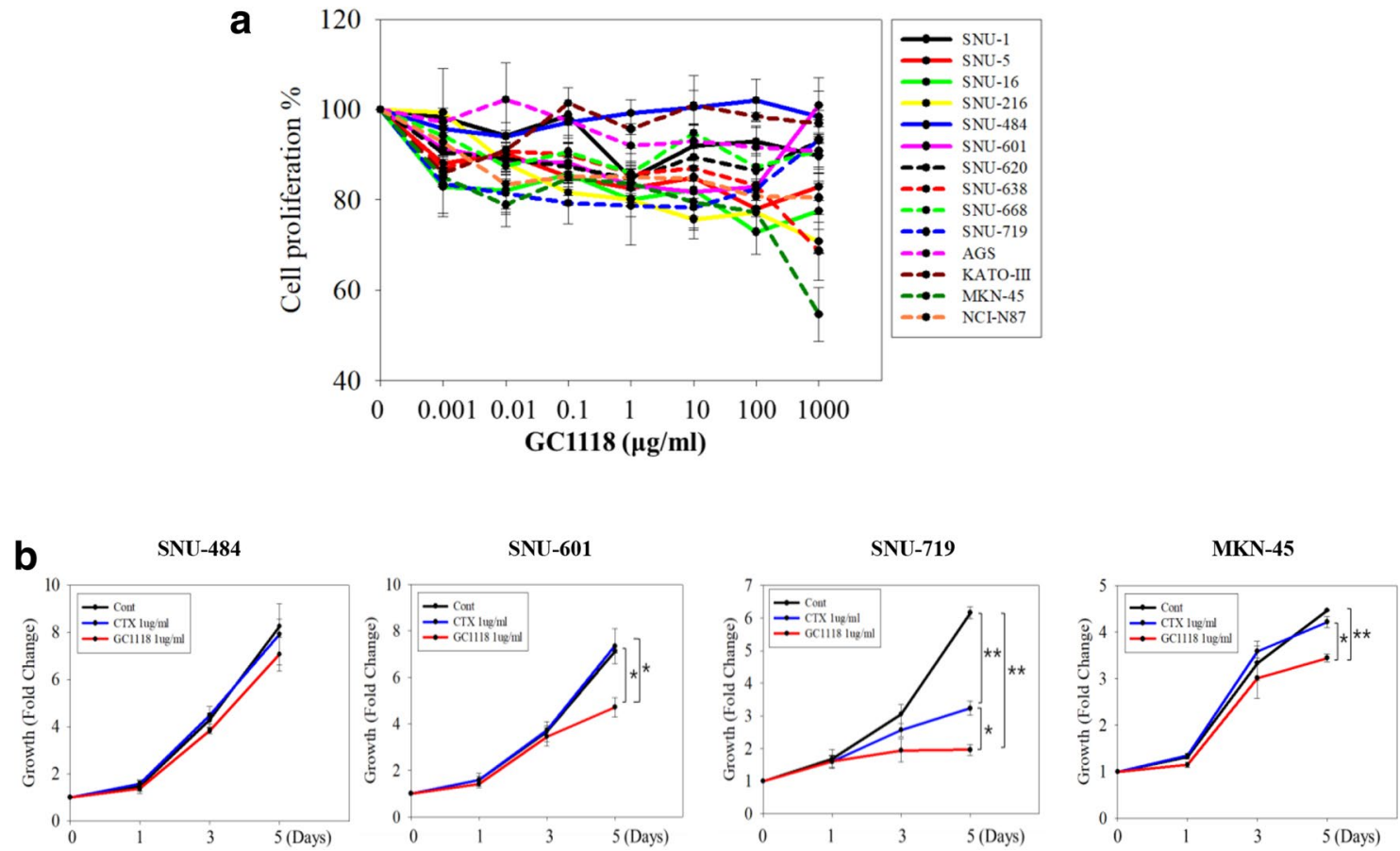

Fig. 1 The anti-growth effect of GC1118 or cetuximab mono-treatment in GC cell lines. a Cell growth was measured after exposure to indicated doses of GC1118 $(0,0.001,0.01,0.1,1,10,100,1000 \mu \mathrm{g} /$ $\mathrm{ml}$ ) for $72 \mathrm{~h}$ utilizing MTT Assay in 14 GC cell lines. Data repre-

sent three independent experiments. b Cell numbers on days 1,3 , and 5 were measured by MTT assay after treatment with GC1118 $(1 \mu \mathrm{g} /$ $\mathrm{ml})$ or cetuximab $(1 \mu \mathrm{g} / \mathrm{ml})$. Data represent three independent experiments. $* p$ value $<0.05$ 


\section{Ligand-induced EGFR activation increased sensitivity to GC1118 in GC cell lines}

Given that the ligand-mediated activation of EGFR is known to facilitate cancer cell survival [10-13], we determined the endogenous levels of the EGFR ligand EGF, TGF- $\alpha$, HBEGF, and amphiregulin in GC cell lines by ELISA (Fig. 2a). Among the 14 cell lines, with their varying levels of ligands, no association between sensitivity to GC1118 treatment and endogenous ligand levels was identified (Fig. 2a). In addition, the basal expression levels of total EGFR and phosphorylated EGFR (Fig. 2b) in GC cells did not correlate with ligand levels. Furthermore, the basal expression levels of total EGFR and phosphorylated EGFR did not predict sensitivity to GC1118 (Fig. 2b).

Next, we examined the anti-proliferative effects of GC1118 or cetuximab following EGFR-ligand stimulation in GC cells. We performed western blotting to determine whether the ligand-stimulated EGFR signaling pathway was inhibited to different extents by GC1118 compared with cetuximab. Under ligand-stimulated conditions, GC1118 dramatically inhibited signaling transduction, while cetuximab failed to block the expression of p-AKT or p-ERK in SNU-216, SNU-601, SNU-719, and AGS cells (Fig. 2c).

\section{GC1118 in combination with cytotoxic chemotherapeutic agents showed more potent antitumor activity than either agent alone}

We evaluated combination treatment of GC1118 and cytotoxic chemotherapeutic agents. Figure 3 a shows the effects of GC1118, cisplatin, and 5-FU alone or in combination on GC cells in a colony-forming assay (Fig. 3a). Although a significant reduction in colony number was observed with GC1118 alone, GC1118 in combination with cisplatin or 5-FU showed more potent anti-proliferative activity in SNU601, SNU-719, AGS, and MKN-45 cell lines (Fig. 3a). The similar effect was also confirmed by migration assay in various cell lines (SNU-216, SNU-601, and AGS), whereby the migration of cells treated with indicated concentrations of GC1118 was more potently suppressed by adding 5-FU or cisplatin (Fig. 3b).

\section{Combination treatment of GC1118 and cytotoxic chemotherapeutic agents disrupts cell growth signals under the EGFR ligand stimulation conditions}

Given the potent effect of GC1118 and cytotoxic chemotherapeutic agents observed in the colony-forming assay and migration assay, we hypothesized whether the combination effect would be maintained following EGFR ligand stimulation. We determined the levels of EGFR pathway signaling markers p-EGFR, p-AKT, and p-ERK in SNU-719 and MKN-45 cells treated with GC1118 plus 5-FU or cisplatin under the EGFR ligand stimulation conditions. Even in the presence of ligand, these markers were downregulated compared with cetuximab plus 5-FU or cisplatin (Fig. 4). The combination of 5-FU and GC1118 under EGF stimulation also induced more PARP and caspase-7 cleavage compared with cetuximab combination groups in SNU-719 cells, but not in MKN-45 cells (Fig. 4).

\section{Xenograft tumor growth was significantly suppressed by GC1118 compared with cetuximab}

To further examine the anti-tumor effect of GC1118, we established a xenograft model in nude mice using AGS (KRAS mutant) cell lines. After 5 weeks of treatment, we observed that cetuximab did not affect tumor growth in AGS cells (Fig. 5). However, GC1118 showed potent antitumor efficacy, regardless of KRAS mutation status. GC1118 showed more significant inhibition of tumor growth than cetuximab $(p<0.05)$.

\section{Discussion}

As mentioned earlier, both cetuximab and panitumumab failed to improve the overall survival in GC patients. Moreover, there is little evidence could support panitumumab better than cetuximab in GC currently. Therefore, we evaluated the efficacy of GC1118 compared with cetuximab in GC cell lines. GC1118 is a novel potent EGFR inhibitor with efficacy in CRC cell lines and xenograft models [7]. Consistent with our results, the authors demonstrated that GC1118 exhibited superior inhibition of proliferation compared with cetuximab, and p-AKT and p-ERK were downregulated to a greater extent by GC1118 compared with cetuximab in the presence of various ligands. We also identified that cotreatment of GC1118 and cytotoxic chemotherapeutic agents strongly inhibited cell migration (Fig. 3b).

Previous studies have evaluated the effects of cetuximab only in KRAS wild-type cell lines [14, 15]. The resistance mechanism to cetuximab shown by many tumor types remains unclear, although $K R A S$ mutation, as well as $E G F R$ mutation and HER2 or MET activation, has been implicated [15-22]. HRAS mutation or upregulated expression of microRNA-100 and microRNA-125b have also been shown to be associated with cetuximab resistance [23, 24].

In the present study, we summarized the main gene alteration status of each cell line (Table 1) from cancer cell line encyclopedia (CCLE). Five of the cell lines, SNU-1, SNU16, SNU-601, SNU-668, and AGS, harbor a KRAS mutation; however, GC1118 profoundly inhibited proliferation in these five cell lines, particularly in SNU-601 and SNU-668 
a

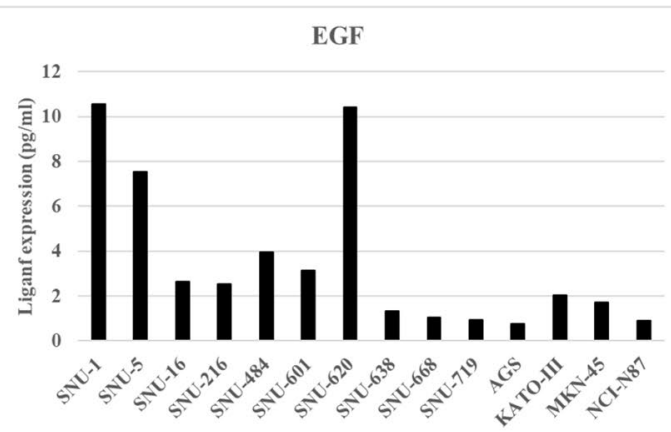

HB-EGF

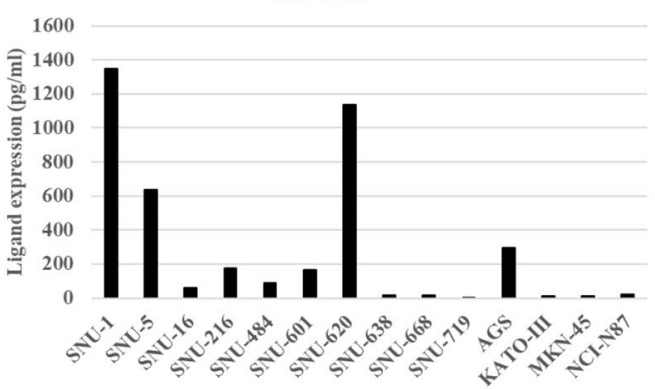

TGF-o

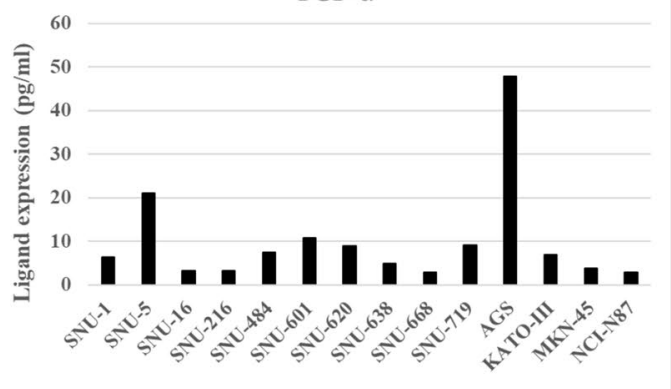

Amphiregulin

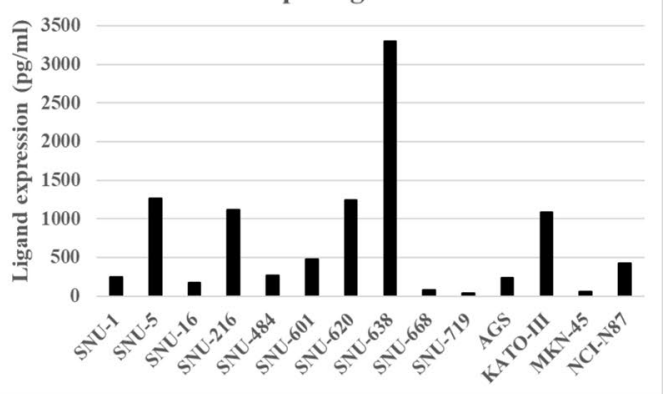

b

C

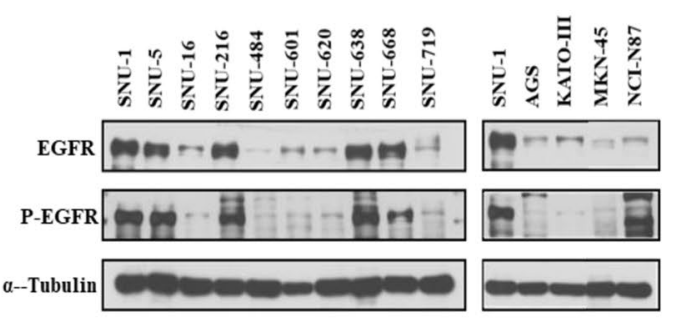

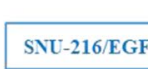

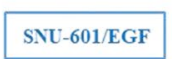

AGS/EGF

SNU-719/EGF
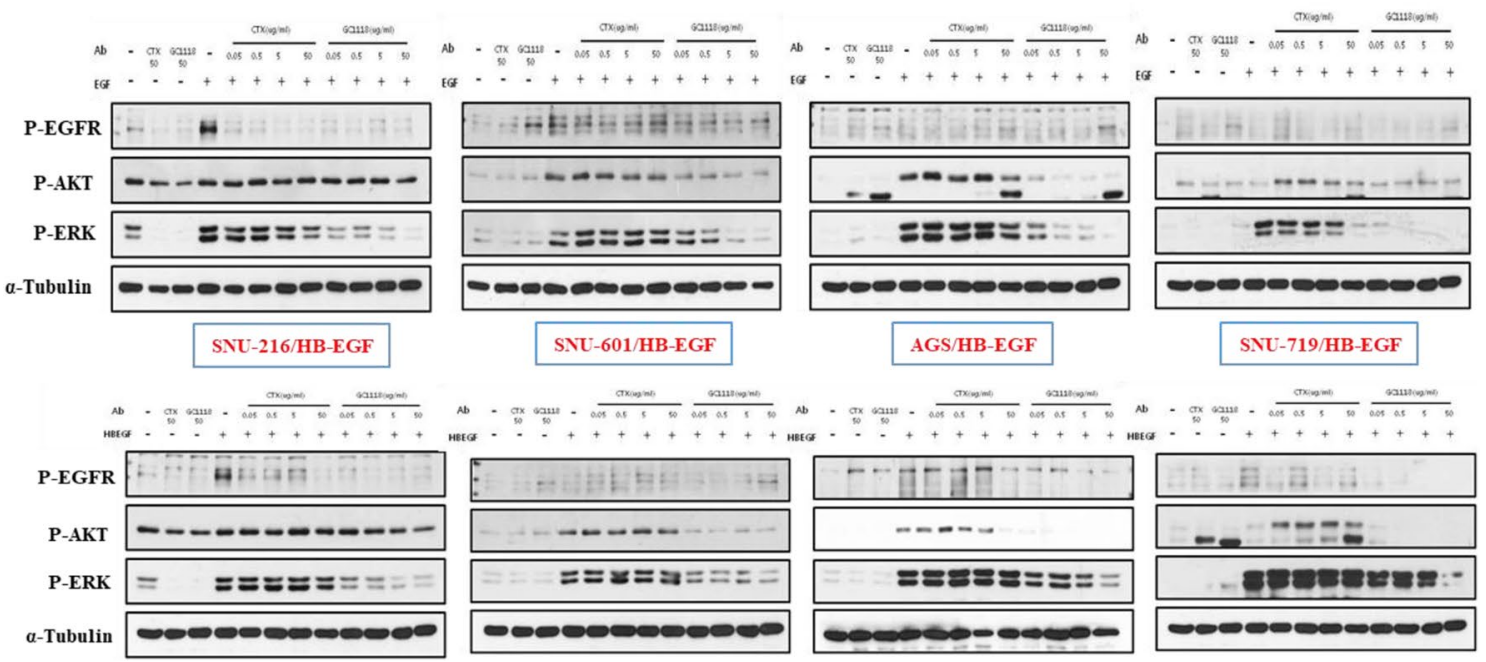

Fig. 2 The effect of GC1118 was evaluated in the presence of ligand stimulation. a The basal secretory levels of EGFR ligands were detected using ELISA kit in 14 GC cells. b Basal expression levels of EGFR and p-EGFR in GC cell lines detected by western blotting. c The indicated cells were treated with GC1118 or cetuximab for $2 \mathrm{~h}$ and then co-treated with or without ligands stimulation for $15 \mathrm{~min}$. After that the main cell signaling was monitored by western blot 
a
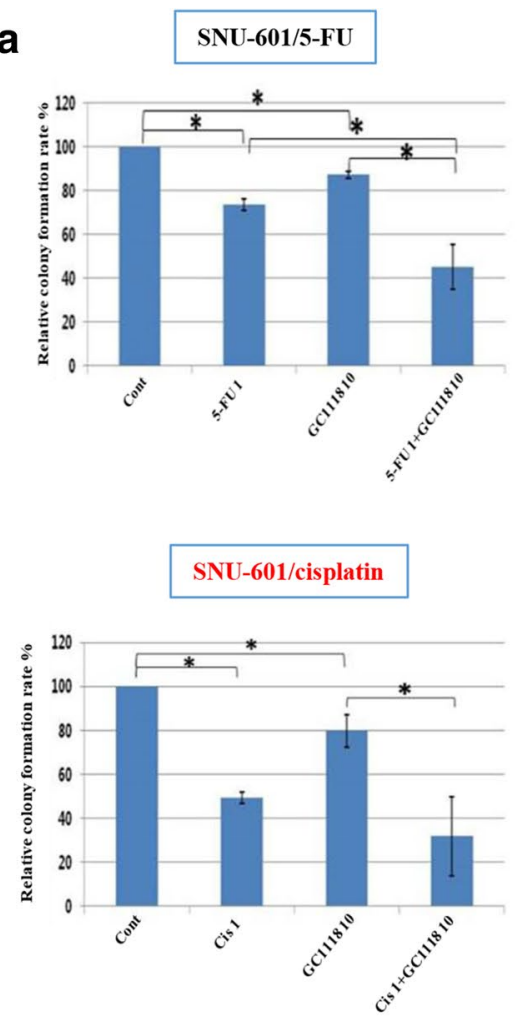

b
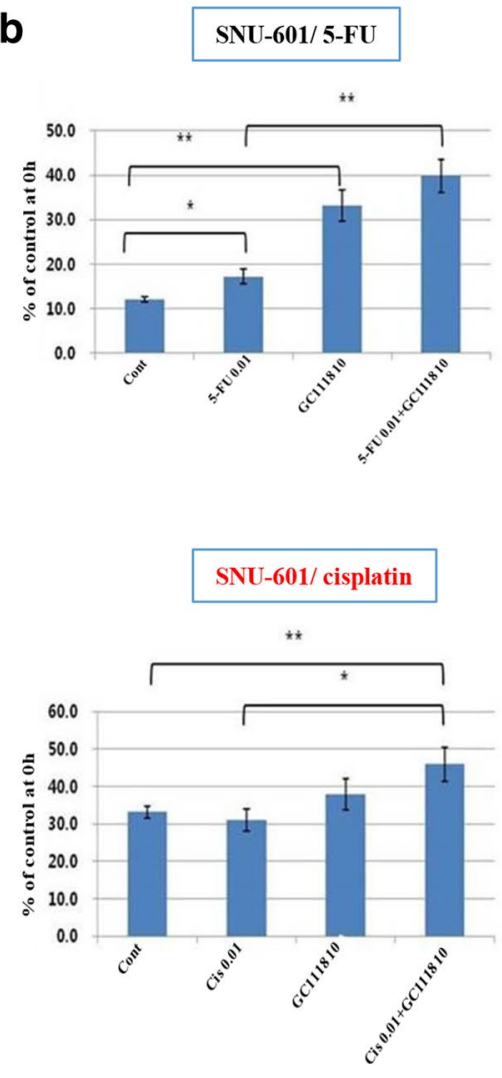
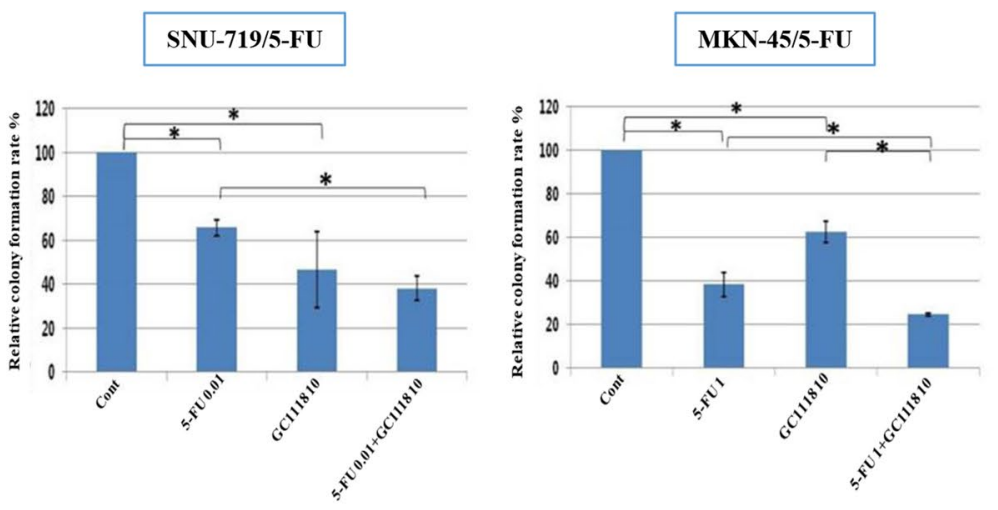

SNU-719/cisplatin
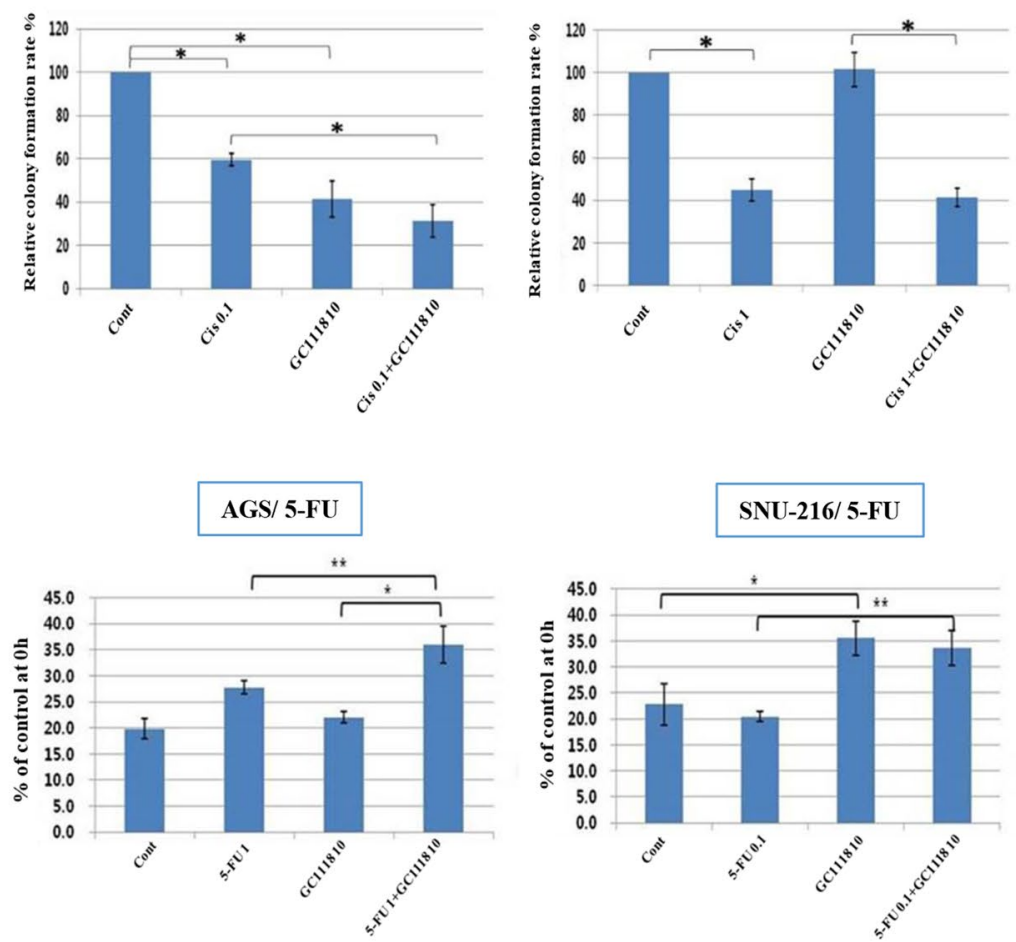

AGS/ cisplatin

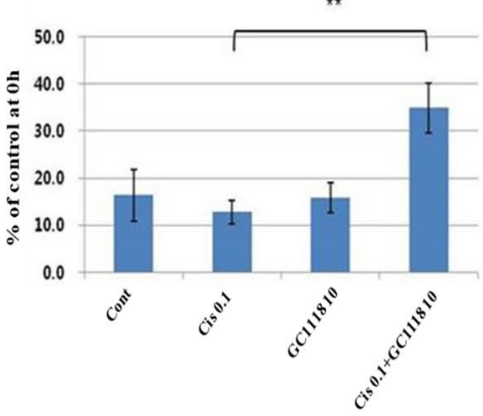


4Fig. 3 The synergistic effect was observed when combined treatment of GC1118 and cytotoxic chemotherapeutic agents. a The indicated cells were exposed to GC1118 alone or cisplatin/ 5-FU or both; 10 days later, colony number was analyzed by Gel Doc system software. Each experiment was conducted three times. ${ }^{*} p$ value $<0.05$. b The cell migration assay was performed in GC1118 or cisplatin/5-FU or both treated cells. The image was captured after $48 \mathrm{~h}$ and the width of the gap in the cell monolayer was measured using ImageJ software. Each experiment was conducted three times. ${ }^{*} p$ value $<0.05$

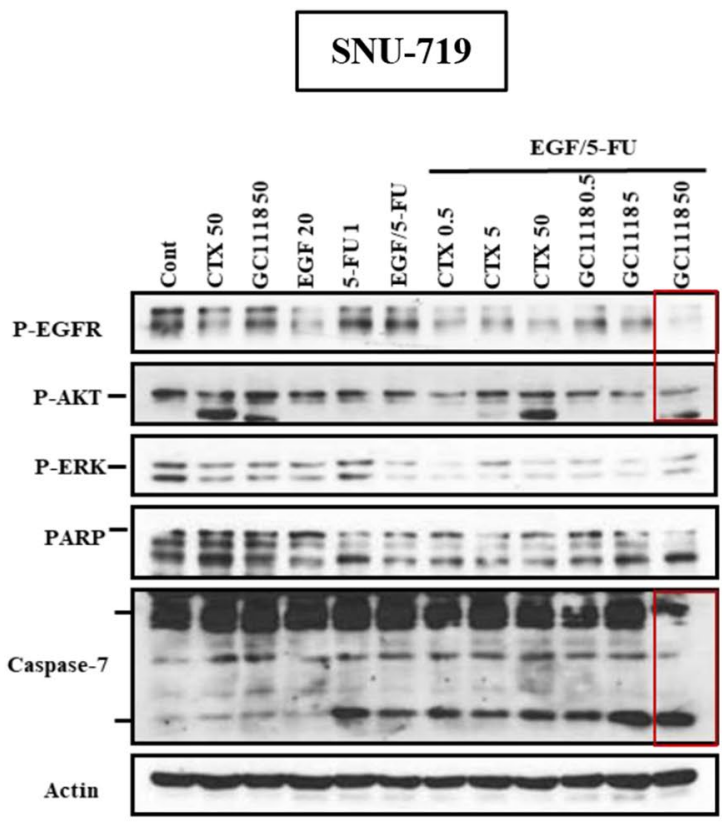

MKN-45

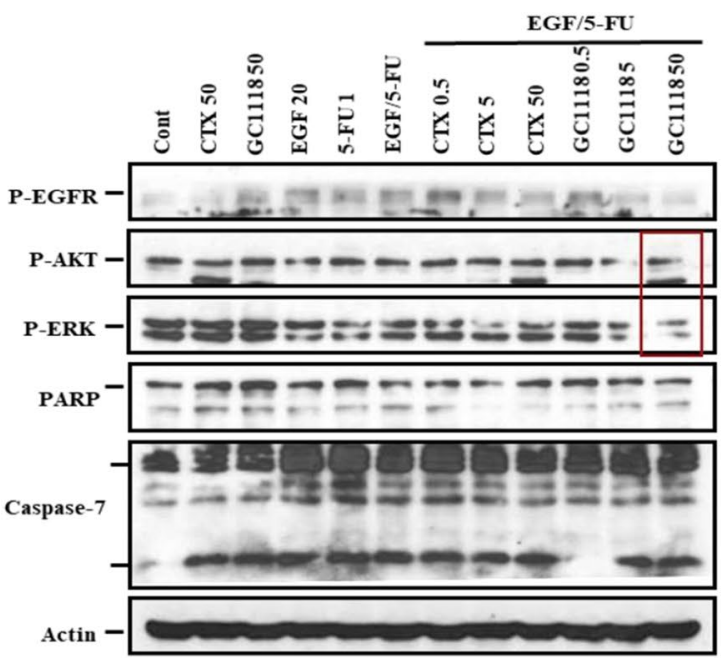

Fig. 4 Combination effect of GC1118 plus cytotoxic chemotherapeutic agents under the ligands stimulation. In this figure, SNU-719 and MKN-45 were incubated in serum-free media for $24 \mathrm{~h}$, treated with GC1118 or cetuximab for $2 \mathrm{~h}$, stimulated by $20 \mathrm{ng} / \mathrm{ml}$ EGF for
(Fig. 1a). An earlier study on GC1118 showed its potent inhibition of high-affinity ligand-induced signaling [7], so two high-affinity EGFR ligands, EGF and HB-EGF, were used in our study. As expected, downregulation of p-AKT and p-ERK was observed in SNU-601, SNU-719, and AGS cell lines following treatment with GC1118 under ligand stimulation conditions (Fig. 2c).

The previous study exhibited that p-EGFR was dramatically blocked by GC1118 than cetuximab in all colon
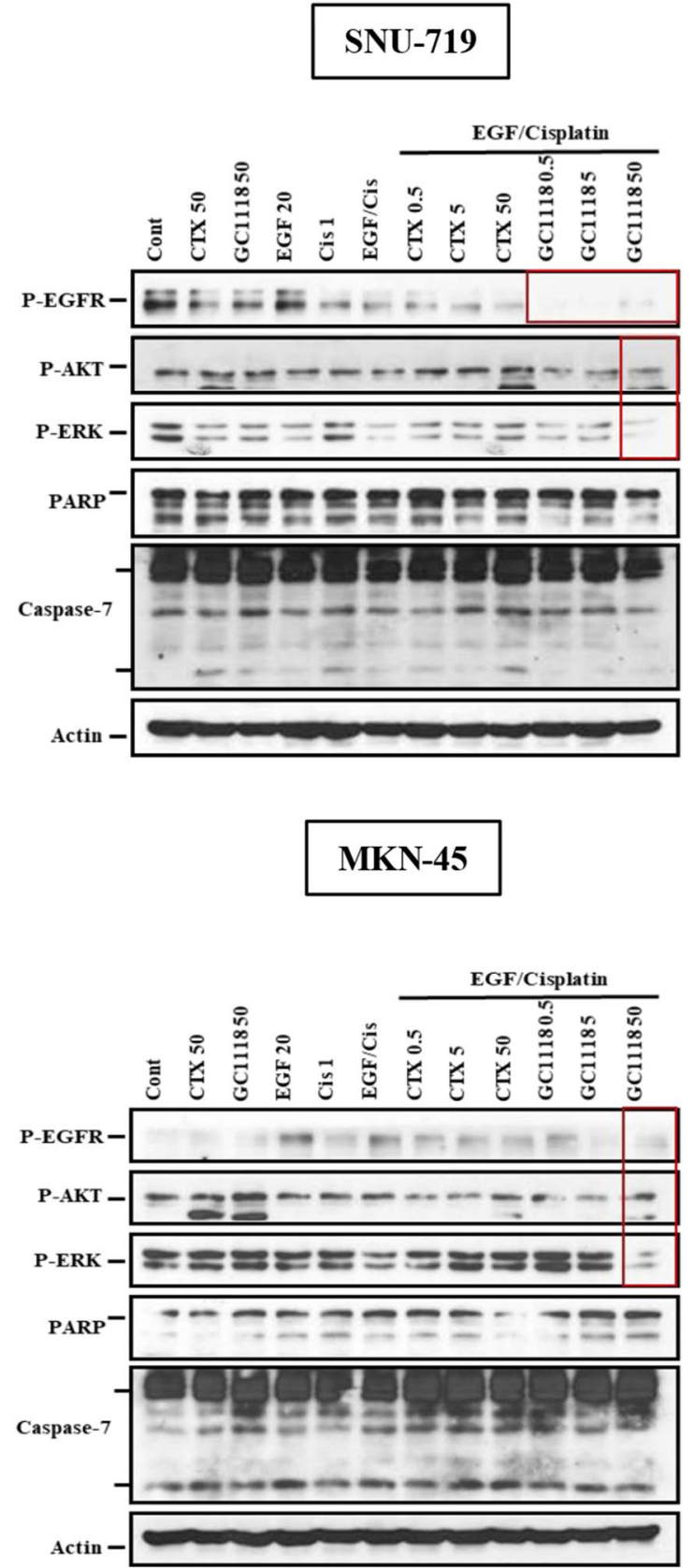

$15 \mathrm{~min}$ and exposed to $1 \mu \mathrm{g} / \mathrm{ml}$ cisplatin or $1 \mu \mathrm{g} / \mathrm{ml} \mathrm{5-FU} \mathrm{for} 24 \mathrm{~h}$. Cell signaling was detected using western blot. The red squares mainly point out the obvious difference between CTX and GC1118 group 
cancer cells, but in GC cells, although we could not observed p-EGFR downregulated by GC1118, both p-AKT and p-ERK were more blocked. Therefore, we speculated that GC1118 may also work on other ERBB family members such as HER 2 or HER3.

In addition, our data showed that combined treatment with GC1118 and cisplatin or 5-FU not only dramatically suppressed colony formation but also inhibited cell migration in SNU-601 and AGS cell lines (Fig. 3). These results indicated that GC1118 has antitumor activity in KRASmutated GC cells whereas cetuximab has not [17]. NRAS mutation has also been identified in the SNU-719 cell line;

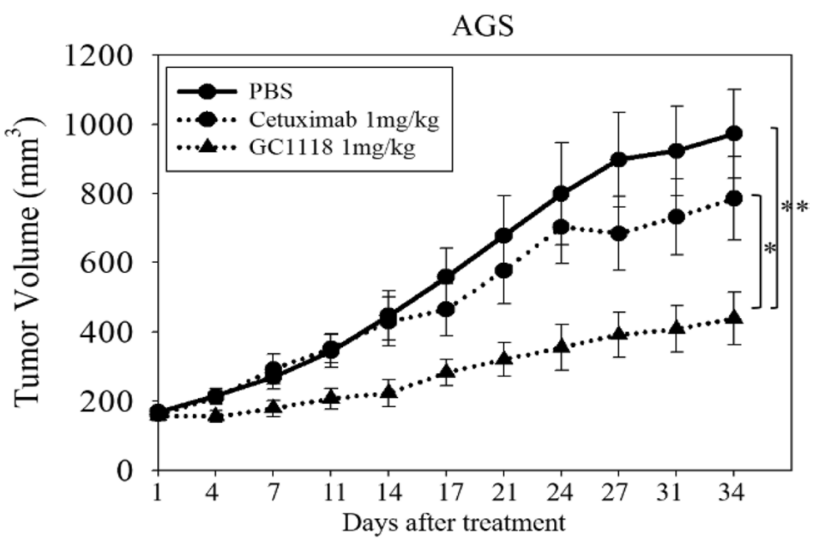

Fig. 5 The anti-tumor effect of cetuximab and GC1118 on xenograft model. Mice were treated with PBS alone, cetuximab $(1 \mathrm{mg} / \mathrm{kg})$, GC1118 $(1 \mathrm{mg} / \mathrm{kg})$ for 5 weeks. Tumor volume was measured every 2-4 days

Table 1 Characteristics of GC cells

\begin{tabular}{|c|c|c|}
\hline Cell lines & KRAS & Other \\
\hline SNU-1 & MT & $\begin{array}{l}\text { Smad } 3 \text { low level, AKT2 mt, STAT3 mt, JAK } \\
\text { deletion }\end{array}$ \\
\hline SNU-5 & WT & MET amplification, TP53 deletion \\
\hline SNU-16 & MT & $\begin{array}{l}\text { FGFR2 amplification, c-myc amplification, } \\
\text { CDLN2A loss, TP53 mt }\end{array}$ \\
\hline SNU-216 & WT & Her2 amplification, TP53 mt \\
\hline SNU-484 & WT & Smad 3 low level, TP53 mt \\
\hline SNU-601 & MT & PIK3CA mt, TP53 mt \\
\hline SNU-620 & WT & TP53 deletion \\
\hline SNU-638 & WT & MET amplification, BRAF mt, TP53 mt \\
\hline SNU-668 & MT & TP53 mt \\
\hline SNU-719 & WT & NRAS mt, PIK3CA mt \\
\hline AGS & MT & PIK3CA mt \\
\hline $\mathrm{MKN}-45$ & WT & $\begin{array}{l}\text { EGFR, c-MET amplification, PIK3CA mt, TP53 } \\
\text { mt }\end{array}$ \\
\hline NCI-N87 & WT & Her2 amplification, TP53 mt \\
\hline KATO-III & WT & \\
\hline
\end{tabular}

however, GC1118 had potent anti-proliferative activity in this cell line and this effect was augmented by combined treatment with cisplatin or 5-FU with ligand stimulation (Figs. 2c, 3a, 4). Our data suggest that GC1118 has antitumor activity even in cells harboring NRAS mutations. Moreover, SNU-216 (HER2 amplification), SNU-5 (MET amplification), SNU-638 (MET amplification/BRAF mutation) and MKN-45 (EGFR/MET amplification) cell lines also responded well to GC1118. Overall, GC1118 showed more potent anti-tumor effects compared with cetuximab in xenograft AGS (KRAS mutant) tumors and this finding was consistent with our in vitro data.

With the ongoing development of immunotherapy, numerous studies of anti-cancer agents, including the effect of EGFR-targeted antibodies on the immune system, are underway $[25,26]$. Interestingly, a recent study showed that cetuximab combined with chemotherapy could induce immunogenic cell death, leading to increased phagocytosis of dendritic cells and immune memory response of T cells [25]. Moreover, in head and neck squamous cell carcinomas, dendritic cell maturation was enhanced by cetuximab or panitumumab, and activated NK cells and cytotoxic T cells were increased via treatment with cetuximab [26]. These results suggested that anti-EGFR antibodies have the potential to modulate the immune response, meaning that further research on the interaction of GC1118 with the tumor microenvironment may be highly beneficial.

In the present study, we found that GC1118 inhibits cell proliferation, migration, and signal transduction more potently than cetuximab in GC cell lines. GC1118 efficacy was observed, regardless of KRAS status. Moreover, antigrowth effect of GC1118 was augmented by combination with chemotherapeutic agents, even under EGFR-ligandstimulated conditions. Our findings, therefore, support the further clinical development of GC1118 for the treatment of GC.

Acknowledgements This study was supported by MOGAM Institute for Biomedical Research fund (Grant No. 06-2013-1580 and 06-2014-3250) and Basic Science Research Program through the National Research Foundation of Korea (NRF) funded by the Ministry of Education, Science and Technology (grant number 2016R1D1A1A09918133).

\section{Compliance with ethical standards}

Conflict of interest The authors disclose no potential conflicts of interest.

Ethical approval All institutional and national guidelines for the care and use of laboratory animals were followed. 


\section{References}

1. Wieduwilt MJ, Moasser MM. The epidermal growth factor receptor family: biology driving targeted therapeutics. Cell Mol Life Sci. 2008;65:1566-84.

2. Yarden Y, Pines G. The ERBB network: at last, cancer therapy meets systems biology. Nat Rev Cancer. 2012;12:553-63.

3. Dokala A, Thakur SS. Extracellular region of epidermal growth factor receptor: a potential target for anti-EGFR drug discovery. Oncogene. 2017;36:2337-44.

4. Kim MA, Lee HS, Lee HE, Jeon YK, Yang HK, Kim WH. EGFR in gastric carcinomas: prognostic significance of protein overexpression and high gene copy number. Histopathology. 2008;52:738-46.

5. Lordick F, Kang YK, Chung HC, Salman P, Oh SC, Bodoky $\mathrm{G}$, et al. Capecitabine and cisplatin with or without cetuximab for patients with previously untreated advanced gastric cancer (EXPAND): a randomised, open-label phase 3 trial. Lancet Oncol. 2013;14:490-99.

6. Waddell T, Chau I, Cunningham D, Gonzalez D, Okines AF, Okines $\mathrm{C}$, et al. Epirubicine, oxalipatin, and capecitabine with or without panitumumab for patients with previously untreated advanced oesophagogastric cancer (REAL3): a randomised, openlabel phase 3 trial. Lancet Oncol. 2013;14:481-89.

7. Lim Y, Yoo J, Kim MS, Hur M, Lee EH, Hur HS, et al. GC1118, an anti-EGFR antibody with a distinct binding epitope and superior inhibitory activity against high-affinity EGFR ligands. Mol Cancer Ther. 2016;15:251-63.

8. Takahashi N, Yamada Y, Taniguchi H, Fukahori M, Sasaki Y, Shoji H, et al. Clinicopathological features and prognostic roles of KRAS, BRAF, PIK3CA and NRAS mutations in advanced gastric cancer. BMC Res Notes. 2014;7:271.

9. Deng N, Goh LK, Wang H, Das K, Tao J, Tan IB, et al. A comprehensive survey of genomic alterations in gastric cancer reveals systematic patterns of molecular exclusivity and co-occurrence among distinct therapeutic targets. Gut. 2012;61:673-84.

10. Shimura T, Yoshida M, Fukuda S, Ebi M, Hirata Y, Mizoshita T, et al. Nuclear translocation of the cytoplasmic domain of HB-EGF induces gastric cancer invasion. BMC Cancer. 2012;12:205.

11. Yotsumoto F, Sanui A, Fukami T, Shirota K, Horiuchi S, Tsujioka $\mathrm{H}$, et al. Efficacy of ligand-based targeting for the EGF system in cancer. Anticancer Res. 2009;29:4879-85.

12. Baek MK, Kim MH, Jang HJ, Park JS, Chung IJ, Shin BA, et al. EGF stimulates uPAR expression and cell invasiveness through ERK, AP-1, and NF-kappaB signaling in human gastric carcinoma cells. Oncol Rep. 2008;20:1569-75.

13. Chakraborty S, Li L, Puliyappadamba VT, Guo G, Hatanpaa KJ, Mickey B, et al. Constitutive and ligand-induced EGFR signaling triggers distinct and mutually exclusive downstream signaling networks. Nat Commun. 2014;5:5811.

14. Hotz B, Keilholz U, Fusi A, Buhr HJ, Hotz HG. In vitro and in vivo antitumor activity of cetuximab in human gastric cancer cell lines in relation to epidermal growth factor receptor
(EGFR) expression and mutational phenotype. Gastric Cancer. 2012;15:252-64.

15. Heindl S, Eggenstein E, Keller S, Kneissl J, Keller G, Mutze K, et al. Relevance of MET activation and genetic alterations of KRAS and E-cadherin for cetuximab sensitivity of gastric cancer cell lines. J Cancer Res Clin Oncol. 2012;138:843-58.

16. Kneissl J, Hartmann A, Pfarr N, Erlmeier F, Lorber T, Keller S, et al. Influence of the HER receptor ligand system on sensitivity to cetuximab and trastuzumab in gastric cancer cell lines. J Cancer Res Clin Oncol. 2017;143:573-600.

17. Misale S, Yaeger R, Hobor S, Scala E, Janakiraman M, Liska D, et al. Emergence of KRAS mutations and acquired resistance to anti-EGFR therapy in colorectal cancer. Nature. 2012;486:532-6.

18. Bardelli A, Jänne PA. The road to resistance: EGFR mutation and cetuximab. Nat Med. 2012;18:199-200.

19. Arena S, Siravegna G, Mussolin B, Kearns JD, Wolf BB, Misale S, et al. MM-151 overcomes acquired resistance to cetuximab and panitumumab in colorectal cancers harboring EGFR extracellular domain mutations. Sci Transl Med. 2016;8:324ra14.

20. Bertotti A, Migliardi G, Galimi F, Sassi F, Torti D, Isella C, et al A molecularly annotated platform of patient-derived xenografts ("xenopatients") identifies HER2 as an effective therapeutic target in cetuximab-resistant colorectal cancer. Cancer Discov. 2011;1:508-23.

21. Kasper S, Breitenbuecher F, Reis H, Brandau S, Worm K, Köhler $\mathrm{J}$, et al. Oncogenic RAS simultaneously protects against antiEGFR antibody-dependent cellular cytotoxicity and EGFR signaling blockade. Oncogene. 2013;32:2873-81.

22. Marzi L, Combes E, Vié N, Ayrolles-Torro A, Tosi D, Desigaud $\mathrm{D}$,et al. FOXO3a and the MAPK p38 are activated by cetuximab to induce cell death and inhibit cell proliferation and their expression predicts cetuximab efficacy in colorectal cancer. $\mathrm{Br} \mathrm{J}$ Cancer. 2016;115:1223-33.

23. Ali M, Kaltenbrun E, Anderson GR, Stephens SJ, Arena S, Bardelli A, et al. Codon bias imposes a targetable limitations on KRAS-driven therapeutic resistance. Nat Commun. 2017;8:15617.

24. Lu Y, Zhao X, Liu Q, Li C, Graves-Deal R, Cao Z, et al. LncRNA MIR100HG-derived miR-100 and miR-125b mediate cetuximab resistance via Wnt/ $\beta$-catenin signaling. Nat Med. 2017;23:1331-41.

25. Pozzi C, Cuomo A, Spadoni I, Magni E, Silvola A, Conte A, et al. The EGFR-specific antibody cetuximab combined with chemotherapy triggers immunogenic cell death. Nat Med. 2016;22:624-31.

26. Trivedi S, Srivastava RM, Concha-Benavente F, Ferrone S, Garcia-Bates TM, Li J, et al. Anti-EGFR targeted monoclonal antibody isotype influences antitumor cellular immunity in head and neck cancer patients. Clin Cancer Res. 2016;22:5229-37.

Publisher's Note Springer Nature remains neutral with regard to jurisdictional claims in published maps and institutional affiliations. 